\title{
Cerebrospinal Fluid Rhinorrhea Secondary to Idiopathic Intracranial Hypertension
}

\author{
Amal Alkhotani \\ Department of Medicine, Umm AlQura University, King Abdulla Medical City, \\ Makkah, Saudi Arabia
}

\section{Keywords}

Idiopathic intracranial hypertension · Cerebrospinal fluid · Rhinorrhea

\begin{abstract}
Idiopathic intracranial hypertension $(\mathrm{IIH})$ is a syndrome of increased intracranial pressure without a mass lesion or ventriculomegaly and normal cerebrospinal fluid (CSF) composition. Patients typically present with a headache and visual symptoms and signs. Here, we discuss a rare presentation of spontaneous CSF leak secondary to $\mathrm{IHH}$ and mild ophthalmic finding that worsened after the cranial repair of the CSF leak.

(C) 2019 The Author(s)

Published by S. Karger AG, Basel
\end{abstract}

\section{Introduction}

Idiopathic intracranial hypertension $(\mathrm{IIH})$, or pseudotumor cerebri, is a syndrome of increased intracranial pressure without a mass lesion or ventriculomegaly and normal cerebrospinal fluid (CSF) composition [1]. IIH is typically a condition affecting young women of childbearing age. Classically, patients present with a headache and papilledema. Spontaneous CSF leak has been reported in a few cases. Here, we discuss a case of IIH in which the patient 


\section{Case Reports in Neurology}

Case Rep Neurol 2019;11:295-298

DOI: $10.1159 / 000503813$

Alkhotani: Cerebrospinal Fluid Rhinorrhea Secondary to Idiopathic Intracranial Hypertension

presented with spontaneous CSF leak and mild papilledema, who subsequently worsened despite continuous treatment after repairing a skull defect.

\section{Case Presentation}

A 35-year-old female patient presented with a 2-week history of unilateral clear discharge from her nostrils. One month before her presentation, she developed a persistent daily headache. It was holocephalic, dull aching, with associated tinnitus in both ears and visual obscuration occasionally. There were no associated neurological symptoms. Her headache responded to painkillers. She had no associated nausea, vomiting or photophobia. Two weeks later, she developed a persistent clear discharge from her right nostril. Her headache improved more than $50 \%$ after the development of rhinorrhea. She was previously healthy and took no medication.

Examination upon presentation revealed a morbidly obese lady with a BMI of 48 . She had a persistent clear discharge from her left nostril. Neurological evaluation showed early papilledema; otherwise the examination was normal.

Brain MRI was also normal. High-resolution skull CT showed an anterior cranial fossa defect. Lumbar puncture revealed an opening pressure of $25 \mathrm{~cm}$ with a normal CSF composition. IIH with CSF leak was diagnosed.

The patient was started on acetazolamide orally. Subsequently, an anterior cranial fossa repair was performed.

She remained asymptomatic on acetazolamide for 6 months following surgery, when she developed a persistent daily headache with visual obscuration and tinnitus. At that time, she had severe papilledema on examination. A repeat lumber puncture showed an opening pressure of $30 \mathrm{~cm}$. A ventriculoperitoneal shunt was later installed to relieve the CSF pressure. She became asymptomatic after surgery.

\section{Discussion and Conclusion}

IIH is a common disorder. The pooled incidence of IIH is 1.20 per 100,000 per year [2]. In young obese women, the estimated incidence is 22 per 100,000 [3]. In total, $90 \%$ of patients with IIH present with a headache that is variable in nature. Table 1 shows the most recent International Classification of Headache Disorders (ICHD-3) [4]. Secondary to elevated intracranial pressure, patients can develop visual symptoms in the form of double vision, blurred vision and transient visual obscuration. The hallmark of IIH is the presence of papilledema on clinical examination, which can be mild or severe depending on the intracranial pressure. It can also be bilateral or unilateral [5]. Patients with IIH should have a full vision evaluation including field assessment. Close ophthalmic evaluation is recommended when IIH is diagnosed. Other symptoms that have been reported with IIH include olfactory dysfunction, pulsatile tinnitus and 6th nerve palsy, which can unilateral or bilateral [4].

IIH is diagnosed by using the updated criteria, which require the following:

1 papilledema;

2 normal neurological examination except for intracranial nerve abnormalities; 
3 neuroimaging: normal brain parenchyma, venous thrombosis excluded;

4 normal CSF composition;

5 elevated lumbar puncture pressure, more than or equal to $25 \mathrm{~cm} \operatorname{CSF}[6,7]$.

Spontaneous CSF leak is a non-traumatic or postoperative complication. It can occur with normal or elevated intracranial pressure. Elevated intracranial pressure can be secondary to the underlying mass lesion, hydrocephalus or IIH [8]. CSF leak can present as CSF rhinorrhea, otorrhea or both. It can result from the disruption of the arachnoid and dura mater in the presence of an osseous defect. The most common sites for a skull base defect are the lateral recess of the sphenoid bone and ethmoid roof [9].

In rare cases, spontaneous CSF leak can present as a manifestation of IIH. Several cases with spontaneous CSF leak have been reported with IIH. In some cases, patients have developed it in a known setting of IIH $[8,10]$. Others state that the first presentation of IIH was spontaneous CSF leak [5, 9]. In some patients, no clear papilledema was initially described, which indicated that the leak was a diversion method to the high CSF pressure.

The management of IIH demands a multidisciplinary approach requiring neurology, ophthalmology involving a dietitian and a neurosurgeon. Most patients with IIH are obese. Weight reduction is thus crucial to address management in those patients.

The cornerstone of management is a reduction in CSF pressure to protect vision. This can be achieved through medical therapy with acetazolamide. In some cases, CSF diversion methods (e.g., a ventriculoperitoneal shunt) are required to decrease persistently elevated CSF pressure or in the presence of vision deterioration [7].

In some cases of spontaneous CSF leak, a combined approach is necessary, which also includes an ENT surgeon. Before repairing the skull defect, treatment targeting the CSF pressure should be initiated.

In the presented case, the patient initially had a headache; however, once she developed spontaneous CSF leak, her headache improved and she had mild papilledema on presentation. Despite medical treatment after the repair of the skull defect, she worsened and developed severe papilledema requiring a CSF diversion. In patients presenting with spontaneous CSF leak after the exclusion of a mass lesion, a lumber puncture with measurements of CSF pressure should be obtained to rule out IIH, even in the absence of papilledema.

In conclusion, for patients presenting with spontaneous CSF leak, increased intracranial pressure should be ruled out. Lumber puncture with pressure measurements should be done. Once IIH is diagnosed, treatment should start before repairing the skull defect. After repair, patients should be followed up closely with ophthalmic examinations, as they may worsen (as illustrated in this case).

\section{Statement of Ethics}

The patient gave a verbal consent to publish her case.

\section{Disclosure Statement}

The author discloses no conflict of interest related to this study. 


\section{Funding Source}

The author did not receive a financial support to publish this article.

\section{References}

1 Bandyopadhyay S. Pseudotumor cerebri. Arch Neurol. 2001 Oct;58(10):1699-701.

2 McCluskey G, Doherty-Allan R, McCarron P, Loftus AM, McCarron LV, Mulholland D, et al. Meta-analysis and systematic review of population-based epidemiological studies in idiopathic intracranial hypertension. Eur J Neurol. 2018 Oct;25(10):1218-27.

3 Goudie C, Burr J, Blaikie A. Incidence of idiopathic intracranial hypertension in Fife. Scott Med J. 2019 May;64(2):62-6.

4 Hoffmann J, Mollan SP, Paemeleire K, Lampl C, Jensen RH, Sinclair AJ. European headache federation guideline on idiopathic intracranial hypertension. J Headache Pain. 2018 Oct;19(1):93.

5 Soin P, Afzaal UM, Sharma P, Kochar PS. Isolated spontaneous cerebrospinal fluid rhinorrhoea as a rare presentation of idiopathic intracranial hypertension: case reports with comprehensive review of literature. Indian J Radiol Imaging. 2018 Oct-Dec;28(4):406-11.

6 Friedman DI, Liu GT, Digre KB. Revised diagnostic criteria for the pseudotumor cerebri syndrome in adults and children. Neurology. 2013 Sep;81(13):1159-65.

7 Mollan SP, Davies B, Silver NC, Shaw S, Mallucci CL, Wakerley BR, et al. Idiopathic intracranial hypertension: consensus guidelines on management. J Neurol Neurosurg Psychiatry. 2018 Oct;89(10):1088-100.

8 Clark D, Bullock P, Hui T, Firth J. Benign intracranial hypertension: a cause of CSF rhinorrhoea. J Neurol Neurosurg Psychiatry. 1994 Jul;57(7):847-9.

9 Rosenfeld E, Dotan G, Kimchi TJ, Kesler A. Spontaneous cerebrospinal fluid otorrhea and rhinorrhea in idiopathic intracranial hypertension patients. J Neuroophthalmol. 2013 Jun;33(2):113-6.

10 Baba M, Tarar 0, Syed A. A Rare Case of Spontaneous Pneumocephalus Associated with Nontraumatic Cerebrospinal Fluid Leak. Case Rep Neurol Med. 2016;2016:1828461.

Table 1. Diagnostic criteria of IIH-related headache according to the International Classification of Headache Disorders (ICHD-3)

A. New headache, or a significant worsening of a pre-existing headache, fulfilling criterion C

B. Both of the following:

1. IIH has been diagnosed

2. CSF pressure exceeds $250 \mathrm{~mm}$ CSF (or $280 \mathrm{~mm}$ CSF in obese children)

C. Either or both of the following:

1. headache has developed or significantly worsened in temporal relation to the IIH, or led to its discovery

2. headache is accompanied by either or both of the following: (a) pulsatile tinnitus, (b) papilledema

D. Not better accounted for by another ICHD-3 diagnosis

IIH, idiopathic intracranial hypertension; CSF, cerebrospinal fluid. Adopted from Hoffmann et al. [4]. 